\title{
Lasers in Ophthalmology: The Basic Principles
}

\author{
JOHN MARSHALL
}

London

In this review I have undertaken a mechanistic approach to the principles underlying interactions between laser light and biological tissues. The surgical potential of the various damage mechanisms are discussed together with the clinical applications in ophthalmology. First however, it may be helpful to briefly relate the history of lasers themselves.

\section{Lasers}

The term LASER is an acronym for Light Amplification by Stimulated Emission of Radiation and is the realisation of a concept postulated by Einstein in 1917 in the course of a theoretical investigation of blackbody radiation. However the problems of developing a satisfactory quantum theory were of sufficient magnitude that the stimulated emission phenomenon was neglected by experimentalists until the early 1950s, although as early as 1946 the process of stimulated emission had been observed, but not recognised in nuclear induction experiments. The use of stimulated emission for microwave amplification was suggested independently by Weber at the University of Maryland, by Basov and Prokhorov in the Soviet Union and by Townes at Columbia University (1953-54). Thus it was in 1954 that the first microwave amplifier or MASER was built and operated. It was not until 1958 that the feasibility of producing stimulated emission in microwaves near the optical regions of the spectrum was realised and a further two years before the first optical maser or laser, the ruby laser, was constructed.

The first continuous wave gas laser, a helium neon system, was demonstrated in 1961 and by 1967 a clinical argon laser was developed specifically for ophthalmic use, becoming commercially available in 1971.
In essence the first laser had a gestation period of 43 years between concept and practical realisation. In contrast, one year after its laboratory inception the first experimental ophthalmic investigation of its surgical potential was published ${ }^{1}$ and unfortunately within five years reports of accidental eye damage had also been recorded. ${ }^{2}$

\section{Laser Surgery}

In laser surgery there are four principal concepts which help in the understanding of the interactions between laser beams and tissues. In sequence these are, transmission, absorption, degradation and reaction. First, the transmission properties of various ocular coats and media must be determined in order to ensure that a given laser wavelength will be transmitted to the required target. Secondly, the absorption characteristics of target sites must be determined in order to ascertain that the incident laser radiation is absorbed within the required system. Thirdly, a knowledge is required of the ways in which the absorbed laser energy is degraded within the target and therefore the ways in which this energy is capable of damaging the target material. Finally, a detailed knowledge is required of the biological reactions within a particular target subsequent to an episode of irradiation. These tissue reactions may be acute and relatively simple to understand such as the instantaneous tearing or rupturing of tissues, or they may be chronic and complex such as those biochemical changes which are thought to ensue after laser treatment of the peripheral retina in diabetes.

\section{Transmission of Optical Radiation}

Optical radiation is somewhat arbitrarily, but conveniently, subdivided into seven wavelength 
regions or photobiological spectral domains which roughly define spectral regimes that elicit similar biological reactions. These wavebands are in order of increasing wavelength; ultraviolet C, (UV-C), 200-280nm; UV-B, 280-315nm; UVA, $315-400 \mathrm{~nm}$; visible radiation, $400-780 \mathrm{~nm}$; infrared A (IRA), 780-1400nm; IRB, $1400-3000 \mathrm{~nm}$ and IRC 3000-10000. The ultraviolet region of the spectrum is adjacent to $\mathrm{X}$-rays and the infrared is adjacent to microwaves. Clearly X-rays are more energetic than microwaves, thus with increasing wavelength the energy of the individual photons of optical radiation decrease.

The various coats and media of the eye can be considered as a series of consecutive spectral filters with each component absorbing exclusive wavelengths within the incident light and thus preventing them from impinging on the next tissue or medium in the pathlength. Essentially all the incident optical radiation at very short wavelengths in the ultraviolet (UV-B and UV-C) and long wavelengths in the infrared (IR-B and IR-C) is absorbed in the cornea and sclera. The lens absorbs strongly in the UV-A, but between 400 and $1400 \mathrm{~nm}$, the eye is specifically adapted to enable incident radiation to penetrate deep within it and fall upon the retina. This waveband (400-1400nm) includes both the visible $(400-780 \mathrm{~nm})$, or that spectal region which initiates transduction within the retina, and the invisible near infrared (780-1400nm). This spectral domain is often referred to as the "retinal hazard region". It represents a particular problem because there is an increase in irradiance $\left(\mathrm{W} / \mathrm{cm}^{2}\right)$ between the corneal epithelium and the retinal which is approximately equal to the ratio of the area of the pupil to that of the retinal image. The pupil is a variable aperture but the diameter may be as large as $5 \mathrm{~mm}$ when maximally dilated in the middle-aged eye. The retinal image corresponding to such a pupil may be between 10 and $20 \mu \mathrm{m}$ in diameter. Thus the increase in irradiance is between $2 \mathrm{x}$ $10^{5}$ and $5 \times 10^{5}$. This increase is implicit in the process of forming an image on the retina by the combined refractive powers of the cornea and lens and is the process which converts the pleasantly warming sensation of midday sunlight upon the skin to the harmful over-exposure of the retina caused by gazing directly at the sun.

\section{The Absorption of Optical Radiation}

The absorption or deposition of laser energy in biological targets takes place at either an atomic or a molecular level. Short wavelength radiation consists of particles or photons whose individual energy may be sufficient to initiate excitation of electrons within target atoms. In contrast, in long wavelength radiation the photons may only be of sufficient energy to initiate vibrational modes between atoms within a molecule. At a more gross level of analysis the absorption process can be roughly divided into two different processes, linear absorption and non-linear absorption. In linear processes the amount of light absorbed by a material is proportional to the amount of light falling upon it. As light per unit area increases the absorbed light is converted into heat and the target tissue temperature increases in proportion to the light per unit area. Effect is then directly proportional to cause the light absorption and temperature increases are termed linear processes. Non linear optical effects occur when the irradiance is so high that the target undergoes changes in its structure and cause and effect no longer are a simple proportional relationship. In linear processes a laser wavelength is chosen that will be absorbed by some pigmented system within the target tissue. The amount of energy absorbed will be dependent upon both biological variables such as; the amount of pigment present, that is its optical density; and the distribution or pathlength over which the pigment is distributed; as well a number of biologically independent variables such as laser energy, pulse duration, and area of irradiation. In non-linear absorption processes the biological variables become relatively unimportant as this mechanism depends upon a high concentration of photons within a highly localised focal plane in a short time period, a nanosecond or less $\left(10^{-9}\right)$, such that the conventional rules of absorption no longer apply. In such instances wavelength is also relatively unimportant provided it can be transmitted to the target. Non linear absorption depends upon the location of the peak power generated by the laser exposure within the focal plane, direct electric field effects, Raman and Brillouim scattering and multiphoton absorption then play a role in the production of damage.

In laser exposure of the retina, linear optical processes predominate. Most visible and near 
infrared radiation is absorbed in the normal retina or choroid in one of five major absorbing systems; melanin, within the retinal pigment epithelium and choroidal melanocytes; haemoglobin, within both retinal and choridal blood vessels; the macular pigment; lipofuscin, in the retinal pigment epithelium and the visual pigments. In some diseased states melanin containing cells may be present in isolation or in aggregates within the neural retina, whilst in others hard exudates (macrophages and lipids), haemorrhages or oedema may be present. Any of these may also absorb or scatter incident radiation.

In the normal retina the melanin in the retinal pigment epithelium is the most ubiquitous of these pigmented systems. The melanin is in the form of fusiform granules and these are usually confined to the apical $5 \mu \mathrm{m}$ of the pigment epithelial, cells. The molecular structure of melanin and its chemical role in these cells is not fully understood, however apart from absorbing light some authorities have suggested that it may also have a role in protecting cells from the radicals generated by light, by its being a powerful electron acceptor or 'free radical sink'. Its light absorbing properties are well known and this $5 \mu \mathrm{m}$ layer absorbs about $40 \%$ of all light entering the eye. The concentration of melanin granules and therefore the optical density varies between individuals and with age, retinal topography and race. In respect of these, highest concentrations are found in the young, in the macula and in negroes. In all cases the spectral absorption is relatively constant with approximately $20-30 \%$ more absorption occurring in the blue green region of the spectrum than in the red.

Lipofuscin is a senescent pigment that progressively builds up within the retinal pigment epithelium with increasing age. It is at its highest concentration in the macula of the elderly and its accumulation is accompanied by a progressive loss of melanin. Little is known of its absorption properties in vivo but it is known to be highly autofluorescent.

The absorption spectra of haemoglobin varies with its degree of oxygenation but in all cases it is a pigment that absorbs more short wavelength radiation than long with peaks at 400,540 and $576 \mathrm{~nm}$. In the normal human retina the majority of vessels are extremely small at less than $10 \mu \mathrm{m}$ in diameter, therefore haemoglobin in such vessels accounts for only a small fraction of the total light absorbed within the eye. However, if the small focused image of a short wavelength laser (e.g. argon $488 \mathrm{~nm}$ ) falls on a major retinal blood vessel the focal absorption within the vessel may be highly significant.

The macular pigment is a yellow pigment found in the inner connecting fibres of cone cells. These fibres collectively form the fibre layer of Henle which is situated between the outer nuclear and outer plexiform layers in the macula. The macular pigment absorbs between 400 and $500 \mathrm{~nm}$ with a maxima at $460 \mathrm{~nm}$. It decreases in concentration with distance from the fovea and again varies beteen individuals and races.

The visual pigments are located on the membrane systems within the outer segments of the photoreceptior cells. The rod cells all contain a single pigment, rhodopsin, which has an absorption maxima around $498 \mathrm{~nm}$. There are three types of cone pigments with maxima at $420 \mathrm{~nm}$ (blue responding), 534nm (green responding) and $564 \mathrm{~nm}$ (red responding). Whilst the visual pigments are not utilised as absorption sites in current surgical procedures absorption of large amounts of blue green light by them during opertive procedures may result in photochemical changes with serious consequences for vision.

Vision radiation that is not absorbed in the preceding five systems may pass on into the choroid where further absorption takes place in the melanin of the choroidal melanocytes and the massive amount of haemoglobin in the choroidal blood supply. Unlike the sparse distribution of the intrinsic retinal circulation the choroid is almost a 'lake of blood', so in all circumstances a significant amount of absorption takes place in the contained haemoglobin. Light that reaches the chorioscleral interface may be reflected back to pass through the entire system for a second time. On this second pass more light is absorbed such that the total reflectance from the human fundus is less than $5 \%$ of the incident light, and virtually all of this reflected light is in the red end of the spectrum.

\section{Degradation of Optical Radiation}

If sufficient radiant energy is absorbed within a biological system the system may lose function 
or physically break down. In all active biological systems repair processes are present, thus radiation induced injuries may be transient or irreversible depending upon the nature, amount and site of the original insult and the time course and extent of the repair processes. In general, repair processes assume increasing significance where damage results from prolonged or chronic repetitive exposures. In surgical applications repair mechanisms are very much a secondary event. The mechanisms by which excessive light induces damage are similar for all biological systems and may involve interactions of ionisation or plasma formation, thermoacoustic or mechanical transients, heat and photochemical processes. A better understanding of these processes has materialised as a result of the urgency for eye and in particular, retinal protection standards caused by the advent of lasers themselves. Thus from studies of laser induced damage to the retina, the following generalisation can be made. At threshold the predominating causal mechanism is broadly related to the pulse duration of the exposure. In order of increasing pulse duration the predominant mechanisms are: picoseconds $\left(10^{-12}\right.$ seconds) to nanoseconds $\left(10^{-9}\right.$ seconds), photodisruption or damage induced by plasma formation; nanoseconds to a few milliseconds $\left(10^{-6}\right.$ seconds), thermoacoustic or mechanical transients; 100 milliseconds to 10 seconds, thermal effects; and in excess of 10 seconds to possibly days or years, photochemical effects. Photochemical effects show a strong wavelength dependency with shorter wavelengths being far more efficient in inducing photochemical damage than longer wavelengths. This is because as previously stated as wavelength decreases photon energy increases and the higher the photon energy the greater the potential for an absorbed photon to induce a chemical change in the absorbing molecule. When extremely short wavelength lasers in the ultraviolet produce damage in tissues with very short pulse durations tilere is a unique damage mechanism termed photoablation. This mechanism results from the very high concentration of extremely high energy photons within the superficial layers of the biological target. The effect comes about because each photon may have an energy in excess of six electron volts and the carbon to carbon bond energy in biological materials is only three electron volts. Clearly any biological molecule that absorbs a photon of such high energy becomes destabilised and biochemical bonds break up. This process is extremely fast with little, if any, thermal component and has been termed photoablation or photon induced molecular decompensation.

Each of the basic damage mechanism has been utilised for carrying out specific surgical procedures in ophthalmology. Proceeding from long to short exposures; photochemical effects have been utilised in so-called photoradiation therapy for the treatment of ocular tumours. Thermal effecis are predominantly utilised for photocoagulation of the retina in such conditions as diabetic retinopathy. Ionising effects have been used for photodisruption of the posterior capsule of the lens subsequent to cataract surgery. Photoablation is currently under experimental assessment for use in a number of different types of corneal surgery.

In summary, the choice of a particular laser for effecting a given surgical procedure is first dependent upon selection of wavelength to ensure that the radiation gets to the desired target and, secondly, selection of damage mechanism to optimise the desired end point. In looking at surgical applications of lasers it is probably easiest to consider these in chronological order of the utilisation of specific damage mechanisms.

\section{Thermal Damage}

If sufficient light is absorbed by a system then the component molecules experience an increased vibration and this is an increased heat content. Thermal injury ensues when enough radiant energy has been absorbed within a tissue to create and sustain, at least for some seconds, a substantial increase in normal tissue temperature. Typically, the increase needs to be in the order of $10^{\circ}-25^{\circ} \mathrm{C}$ to cause permanent harmful changes within exposed cells. In most clinical laser irradations local temperature rises far exceed this figure and cells within areas of irradiation show severe burn characteristics such as gross vacuolation and coagulation or denatured protein components. In the photoreceptor outer segments such thermal damage is seen as a vesiculate disorganisation of the disc membranes, together with diffuse areas of increased electron density. Thermal damage is 
usually confined to a limited area extending either side of the energy absorbing layer and centred on the irradiating beam. As heat is conducted away from the irradiated area of the absorbing system both during and after the exposure, the area over which the radiation is delivered is a fundamental factor in determining the threshold for thermal injury. In other words for thermal injuries the irradiance required to produce a given amount of damage decreases with increase in absorbed image size. The absorbed image size is the same as the image size when beams are focused on extended absorbing systems that are larger than the crosssectional area of the irradiating beam, e.g. the pigment epithelium. However if the absorbing system is very much smaller than the irradiating beam e.g. a $7 \mu \mathrm{m}$ retinal capillary irradiated by a $500 \mu \mathrm{m}$ argon laser spot, then the effective thermal image and associated heat flow from the vessel may be negligible as in this case the absorbed image is a cylinder maximally only $500 \mu \mathrm{m} \times 7 \mu \mathrm{m}$ in size. Similarly the depth of tissue over which absorption takes place will affect the magnitude of the local temperature rise. Thus for a given optical density and a given exposure, the shorter the pathlength over which energy is absorbed the greater the power density and the higher the focal temperature. A clear example of this was demonstrated by the Munich group, who showed that whilst the total energy absorbed in the choroid far exceeds that in the pigment epithelium, the energy absorbed per unit length is far greater in the latter and therefore the highest temperature is generated in this tissue. Heat flow is also modulated by other factors such as the proximity and dimensions of adjacent blood vessels and the fluid dynamics of the contained blood.

\section{In summary:}

1 All clinical exposures using argon, krypton or dye lasers result in thermal damage to the retinal pigment epithelium regardless of the primary target.

2 Argon exposures placed on major retinal vessels result in thermal damage to the vessels and surrounding tissues only when the absorbed image size is relatively large. In practice this means when the vessel diameter approaches that of the image size of the irradiating beam.
3 Argon irradiation of capillaries does not produce a large enough thermal image to result in significant amounts of tissue damage.

4 Krypton irradiation of either large or small vessels does not result in sufficient absorption to induce tissue damage unless exceptionally high irradiations are used on major vessels.

5 Argon blue green irradiations in the macula may result in damage to the inner retinal layers as a result of absorption of argon blue by the macular pigment; such damage does not occur with krypton or argon green only.

6 All clinical exposures with argon, krypton and dye lasers result in thermal damage to the retinal aspect of the choroid. For any given irradiance krypton damage to the choroid is more severe than that of argon and occurs at deeper levels (with respect to the retina) within the choroid in many instances resulting in choroidal oedema and in some cases choroidal haemorrhages.

All laser induced damage is a function of both the initial events caused by absorption and degradation of the incident radiation and a series of secondary events or tissue responses. The initial tissue responses could be described as biological amplifications as they usually extend outside the area of irradiation. Two simple examples of this may be cited. First, low energy laser irradiation of the retinal pigment epithelium results in heating of the melanin granules and thermal transients passing into the pigment epithelial cell, the superficial elements of the choroid and towards the tips of the overlying photoreceptor outer segments. If a critical volume of the outer segments are damaged by thermal transients the whole cell may die. This is clearly demonstrated in the macula where laser damage to the tips of the cone outer segments result in degenerative changes throughout the cells $500 \mu \mathrm{m}$ length. The demonstration of the secondary nature of these degenerative changes is made more dramatic by the fact that the inner connecting fibres of these cells, in the fibre layer of Henle, run at an oblique angle away from the path of the incident radiation and presumed thermal transients. A second example of biological amplification may be seen in those cases where argon laser irradiation has fallen upon a large retinal vessel. In this case thermal transients emanating from the energy dissipated within the vessel reach the 
adjacent nerve fibres. Within a few hours a blockage of axonal flow is noted within the affected fibres giving rise to the so-called 'cytoid bodies. These bodies are always situated on the side of the lesion proximal to the optic disc, and represent axonal swellings due to aggregations of organelles resulting from interruption of axonal flow. If the causal injury is sufficiently severe the proximal portions of affected axons subsequently degenerate throughout their length. The distal portions attached to the ganglion cell bodies degenerate at a much slower rate.

Whatever the site or nature of the insult the early reaction sequence of absorption, degradation, tissue reaction or biological amplification is always followed by attempts at repair. In the retina the response to injury is usually somewhat incorrectly described as one of slow degenerative decay. This is a little unfair because although damaged neural elements are not replaced, retinal damage is relatively quickly and neatly contained. The first stage of the repair process subsequent to laser injury is seen in the presence of sub-retinal macrophages in the interreceptor matrix within a few hours of exposure. The origin and path of entry of these cells into the retina is unknown but they appear so quickly and in such large numbers that at least some must be blood borne. Within a day or two the undamaged pigment epitherlial cells adjacent to the irradiated area undergo changes such that some round-up and bud-off Bruch's membrane whilst others flatten and slide along it in an attempt to recover the area denuded by loss of damaged cells. The wandering pigment epithelial cells really carry out their normal role as photoreceptor specific macrophages but in this case in a relatively strange location, whilst the flattened cells on Bruch's membrane are attempting to perform another of their functions in re-establishing a blood retinal barrier. After some initial controversy in the literature there is now general agreement from autoradiographic studies that retinal pigment epithelial loss during photocoagulation is made good by cell division. Interestingly, in humans, these cells seem to behave rather like the corneal endothelium in that we seem to be born with our complement of cells and throughout life as cells die the population decreases and gaps are prevented by an increase in size of remaining cells. However both epithelium and endothelium have the capacity to divide and do so in vitro in tissue or organ cultures and in vivo in response to acute trauma.

Autoradiographic studies have also demonstrated other cell populations undergoing cell division in both the choroid and neural retina. As previously stated, for any given irradiance, krypton lasers result in more damage to the choroid than argon. However, in all clinical exposures both systems result in some damage and usually temporary closure of the choriocapillaris in the area of irradiation and in the case of both argon and krypton dividing fibroblasts and melanocytes are subsequently seen.

In the neural retina analysis of autoradiographs of retinal histological sections demonstrate that the predominant population of dividing cells was that of retina glia, both Müllers fibres and accessory glia. Such observations further support the concept that neuronal loss in the retina is always accompanied by a repair process that involves glial proliferation. For most laser lesions whatever the intended target or treatment regime, i.e. peripheral ablation, disciform or macular grid, the end result is a loss of photoreceptor cells and a scar involving the interdigitation of proliferated glial cell elements with those of proliferated pigment epithelial cells. In some instances where argon laser irradiation has fallen on a major retinal vessel, endothelial cell proliferation is seen and interpreted as an immediate response to replace damaged cells and re-establish the blood retinal barrier properties of the vascular endothelium.

\section{In summary:}

1. All clinical laser exposures result in some degree of photoreceptor cell loss.

2. All clinical laser exposures result in a glial scar.

3. Focal cell division occurs in response to primary damage in both argon and krypton exposures in (a) the pigment epithelium; (b) the endothelial cells of the choriocapillaris; (c) the choroidal cells near the retina such as fibroblasts and melanocytes.

4. Focal cell division also occurs in response to primary damage in (a) the endothelial cells of deep (with respect to the retina) choroidal vessels in krypton exposures; (b) the 
endothelial cells of large retinal vessels after argon irradiation.

5. As a secondary response to neuronal cell loss focal cell division occurs in retinal glia in both Müllers fibres and accessory glia.

6. As a further secondary response retinal capillary endothelial cells undergo division initially over the area of irradiation and then diffusely radiating away from this area. This secondary response may result from stimulatory chemical disturbances initiated by primary changes in the pigment epithelium such as, cell death, barrier dysfunction, or cell proliferation.

\section{Clinical Applications of Thermal Damage}

Therapeutic retinal photocoagulation has been practised over the past forty years. Initial experiments were carried out in the late 1940s by Meyer-Schwickerath and concurrently by Moran-Salas. The initial studies used focused sunlight but the introduction of the Xenon arc photocoagulator in 1956 provided both a more reliable and a more controllable source of intense light. The ruby laser was used during the early 1960 s to treat a number of retinal conditions. However, although it was found to be acceptable in producing chorioretinal adhesions for treating full-thickness retinal breaks, it was less successful in the treatment of proliferative diabetic retinopathy.

This lack of success of the ruby laser in treating retinal vascular conditions was originally attributed to the inability of red light to be absorbed within the haemoglobin in retinal capillaries. More recent studies on the biophysics of beam tissue interactions now indicate that the wavelength of the emitted light was not of prime importance but that the pulse duration of the early ruby systems was too short to initiate an adequate photocoagulation without the risk of haemorrhage.

The increasing risk of haemorrhage with decreasing pulse duration is now well established and is known to be related to the speed at which tissue fluids are converted into gas. Above threshold the more rapidly the tissue acquires thermal energy the more rapid the phase transitions and volumetric changes. In the outer retina if thermal episodes are sufficiently fast then the associated volumetric changes may result in bulk physical displacement of tissue with tearing or rupturing of structures such as Bruch's membrane or the choriocapillaris.

To some extent the initial misconceptions concerning the relative importance of wavelength led to the development of the blue green continuous wave (cw) argon laser photocoagulator by L'Esperance. This became commercially available in 1971 and was in widespread clinical use by 1975 . However the advent of this system changed both the wavelength and time domain of retinal exposures simultaneously and this induced some confusion in relation to factors underlying the undoubted success of this instrument. A number of clinical trials have since been undertaken to assess the efficacy of photocoagulation as a therapeutic procedure and the efficiency of the argon laser as a radiation source. The trials have demonstrated that panretinal photocoagulation is an effective procedure in relation to proliferative diabetic retinopathy and that grid treatment is beneficial in treating diabetic cystoid macular oedema. Less conclusive results have been found in relation to the vascular complications of senile macular degeneration where early beneficial results were not maintained.

One of the complications of using argon blue green lasers for treating macular conditions arises because of the presence of the yellow, luteal pigment within the inner neuronal layers of the central retina. The early argon photocoagulators emitted more than $70 \%$ of their radiation in the blue $(488 \mathrm{~nm})$ and this is close to the peak absorption of the luteal pigment (460nm). Many early treatments of the macular region using such systems resulted in nontherapeutic damage to the inner retinal layers and a resultant loss of vision.

More recently this problem has been addressed in two ways, first by the production of a new generation of argon lasers in which the blue emission is precluded from entering the eye by specialised optics within the laser and second by the advent of a red light emitting laser, the krypton laser. Further trials have demonstrated that krypton red and argon green (blue green) are equally effective in relation to treating proliferative diabetic retinopathy.

In the last few years, the advent of the cw tunable dye laser with the ability to select the wavelengths of emission throughout the visible spectrum has been somewhat disappointing in 
terms of the ability to use specific wavelengths to selectively coagulated precise targets within the neuronal matrix of the retina. It has, however, further demonstrated that when a therapeutic effect is dependent upon a thermal damage mechanism, resulting from absorption and degradation of energy within the pigmented systems of the outer retina and choroid then the wavelength of the source is relatively unimportant outside the distribution of the macular pigment. This is because all the absorbing systems in this region are confined within 300-500 microns of each other. By the time sufficient energy has been deposited to elevate the temperature of any absorbing system above that required for protein denaturation and photocoagulation, elevated temperature profiles will have been conducted over sufficient distances that they will have traversed elements of each of the other absorbing systems and hence specificity will be lost.

Shortly after the advent of the argon laser's use for retinal procedures it was also employed to treat the trabecular meshwork in eyes suffering from glaucoma. The idea in these procedures was that by depositing a small amount of energy in the thermal domain on the corneal aspect of the trabecular meshwork, the tissue reaction was such that the trabecular beams of the meshwork became stretched and the apertures between them more open. Workers in this field postulate that this will increase aqueous outflow and thereby reduce the intraocular pressure.

In the most recent series of procedures using photocoagulation lasers have been coupled with fine optical fibres $(200 \mu \mathrm{m}$ in diameter $)$ which are then passed through the wall of the eye in order to convey the laser radiation directly to internal structures of the globe. These lasers with optical fibre connections are termed endolasers and their prime use is to photocoagulate peripheral retina subsequent to vitrectomy surgery. Endolasers are also used to seal off small blood vessels which may haemorrhage as vitreoretinal membranes are dissected. Currently a number of investigators are also utilising fibre optic delivery systems with various miniature lens assemblies in order to photocoagulate ocular tissues through the sclera. The underlying concept is that using certain wavelengths, mainly infrared, and appropriate lenses, energy may be brought to a deep focus from outside the wall of the eye on to structures within. The particular area of interest in this type of surgery is for the destruction of the ciliary body in relation to certain cases of glaucoma.

A further development in the field of clinical applications of thermal damage is the use of diode lasers as light sources. These devices are small $(3 \mathrm{~mm} \times 1 \mathrm{~mm})$, require no cooling, currently give output powers in the order of $1-2 \mathrm{~W}$, and can be driven by batteries. Such micro or personal laser photocoagulators may well be the future instruments for retinal surgeons.

\section{Ionizing Damage}

By the mid seventies a new laser modality was seen in the commercial development of short pulsed YAG lasers. Initially, systems were either Q-switched (with nanosecond pulse durations) or mode locked (with picosecond pulse durations). These lasers operated with an emission wavelength at $1064 \mathrm{~nm}$ in the near infrared and therefore have transmission properties whereby they can be brought to a focus anywhere within the eye. The short pulse duration and high peak powers were such that providing a discreet focal plane is defined, these lasers produce such high irradiances that at the point of laser beam focus a small volume of material is ionised. That is the material disintegrates into a collection of ions and electrons called a plasma. The net result of this ionisation process is that targets do not have to be pigmented and all the operator has to do is to focus at his desired site of disruption and then the enormous peak powers concentrated within the focal point will ensure that the target disintegrates.

The sequence of damage mechanisms attendant upon the high focal irradiances generated by short pulsed lasers are as follows; laser pulse, plasma production, shock wave, plasma growth vaporised target material, cavitration bubble. ${ }^{3}$ As well as these processes the ionisation may also result in the production and high speed movement of loose particles of tissue debris, transient high temperatures, emission of optical radiation from the plasma including ultraviolet radiation, the production of free radicals and electromagnetic field stress across cell membranes.

All the physical parameters associated with 
short pulse YAG exposures are difficult to comprehend in terms of the extremes of their dimensions. As examples, I will highlight a few of these with some suitable comparisons. Exposure times are typically a few nanoseconds or picoseconds long. If a picosecond were as long as a second, a second would be thirty-two thousand years. The speed of light is 186,000 miles/second and the distance between the earth and the moon is 240,000 miles, so light coming from the moon takes 1.3 seconds to reach the earth. In a nanosecond light travels about one foot and in a picosecond about $300 \mu \mathrm{m}$. The plasma temperature may instantaneously exceed $10,000^{\circ} \mathrm{C}$ or more than twice that of the surface of the sun. The peak powers in the focal point of the beam may be in the order of giga or terawatts, that is roughly equivalent to the entire output of five major power stations instantaneously discharging their loads into the area of a shirt button. Given this totally unphysiological series of parameters it is not surprising that extensive research has been carried out on the potential intracellular complications of such exposures.

Starting with the initial phenomenon of plasma formation, although the nature of the target material is relatively unimportant it will result in differences in the threshold of plasma creation and show marked differences between Q-switched and mode locked systems. In general the energy levels for plasma production are higher for Q-switched lasers than for mode locked systems and plasma threshold energies decrease with the molecular density of the target. Hence less energy is required to produce a plasma in a solid than a liquid and less in a liquid than a gas. The differences in plasma formation between the two types of YAG lasers relates to the stage immediately before ionisation, called pre-ionisation. With Qswitched systems the irradiance $\left(\mathrm{W} / \mathrm{cm}^{2}\right)$ is sufficient to cause temperature increases in impurities within the target and thereby to produce starting electrons by a process of thermionic emission. In contrast, with the higher irradiances achieved in mode locked pulses, electrons within the focal plane of the beam may simultaneously absorb several photons (multiphoton absorption). Once starting electrons have been produced the ionisation process probably proceeds by an "electron avalanche" with each successive free electron absorbing a photon and together possessing enough energy to ionise an atom. Further electrons are released and repeat the process.

In practice many clinicians, especially those in training, work at energy levels above threshold and under such circumstances a number of potential complications can arise, which include plasma growth, plasma wander and multiple plasma. The concept of plasma growth is obvious but plasma wander and multiple plasmas perhaps need further discussion. Plasma wander is the real location of the plasma in relation to the desired location defined by the focal point determined by the operator. The random micromovements of successive plasmas produced by a beam maintained at a constant focal point probably occur as a result of initial plasma seeding by target impurities near the focal point. Wander is a problem when treating close to a structure that should not be damaged, such as trying to treat a posterior capsule without damaging an intraocular lens (IOL). This sort of treatment regime also suffers from plasma growth and multiple plasmas because, as plasmas grow they develop back towards the operator and multiple plasmas if generated, will occur between the principal focus and the operator. In practice, it is always preferable to focus on the desired target and then to move the focal point just a little way behind the target.

The pressure waves following plasma generation are very similar to those associated with an underwater explosion. Shock waves are pressure waves moving faster than the speed of sound. In clinical practice shock waves are probably generated by the plasma but do not propagate at hypersonic velocities for more than $100 \mu \mathrm{m}$ before decelerating and continuing as acoustic waves. Pressure waves move away from the plasma site in a spherical fashion. This is in contrast to the movement of debris or particles which tend to move in a roughly linear pattern either upstream or downstream to the laser pulse. Detailed measurements of this latter phenomenon have shown that it is associated with plasma growth and this tends to be linear and as previously stated extends back towards the operator. Recent studies have shown that pressure waves are not likely to be the major cause of damage to remote sites such as that seen in corneal endothelial cells subsequent to posterior capsulotomy. Currently this damage is 
considered to arise through one of the following mechanisms; strike by debris or particles moving within a pressure wave front; damage from toxins such as free radicals carried in high concentration within the bubbles; damage resulting from dissipation of the stored energy within the bubble as the bubble cavity collapses. A recent study on the latter phenomena has shown that in liquids without close boundary conditions, or when bounded by elastic borders, bubbles may remain almost stationary, in contrast when bordered by inelastic boundaries they will migrate to the boundary and collapse. In the eye this means that they rarely migrate to the elastic capsule or cornea, but may well collapse and burst on the surface of the intraocular lens. If the bubbles are large enough, bubble collapse may pit the surface of the IOL.

It should be emphasised, however, that most damage to intraocular lenses results from plasma or shock wave related processes due to inappropriate focusing by the operator or the use of too much energy resulting in plasma growth or multiple plasmas.

\section{Clinical uses of Ionisation Damage}

Short pulse YAG lasers were initially used to treat the secondary complication of extracapsular cataract surgery. ${ }^{4}$ Unfortunately in many cases of extracapsular surgery twelve to twenty-four months after surgery a cataractouslike phenomenon is seen to develop in the posterior portion of the capsule. By focusing on this opacity with the YAG laser such opacities could be dispersed as a result of small holes being blown in the capsule followed by capsular ripping. This whole procedure takes just a few minutes, the eye is not opened surgically and the patient is treated as an out-patient. YAG laser capsulotomy has revolutionised modern cataract surgery. With detailed clinical studies it became apparent that mode locked systems were not as efficient for this procedure as Q-switched systems and therefore the former have almost universally fallen out of commercial production.

The ionisation process of YAG laser impacts have also been used to disrupt membranes within the vitreous; and to blow small holes through the iris, either in cases where the natural pupil has been occluded or in cases of closed angle glaucoma. By blowing a small hole in the peripheral iris, fluid can pass through and into the drainage system thereby lowering the intraocular pressure.

Some limited exposures have been made in the thermoacoustic time domain using pulse dye lasers, again to blow small holes in the peripheral iris to alleviate the symptoms of closed angle glaucoma. Pulsed dye laser systems have not, however, received a great deal of acclaim within the ophthalmic community.

\section{Photochemical Damage}

This is the type of light-induced damage that all individuals charged with clinical care of eyes should now at least be aware of, as it is produced by absorbed energy levels far below those that are required to produce thermal damage. In this case the damage results from photochemical reactions in which single photons have sufficient quantum energy to convert individual absorbing molecules into one or more different molecules (free radicals) which may be toxic to the cell. All photochemical injury mechanisms demonstrate a reciprocity relationship between irradiance (dose rate) and exposure duration. That is to say, a constant radiant exposure or dose is required to elicit the response over a wide variation of exposure durations up to those at which biological repair times come into play. As photochemical damage depends on the absorption properties of specific macromolecules, such damage tends to be highly wavelength specific and photobiologists refer to the relative spectral effectiveness of radiation in eliciting any particular biological effect in a particular tissue as an action spectrum. Action spectra usually have extremely steep slopes and it should be emphasised that routinely extrapolating biological injury data between wavelengths or tissues or assuming that apparently smooth curves have no fine structure is to be avoided in analysing such damage. Most photochemical action spectra drop off steeply in the long wavelength end of the spectrum. This is because the yield of photochemical reaction-products is proportional to photon flux and each individual photon must have sufficient energy required for the reaction. At the long wavelength end of the action spectrum the energy of a single photon coupled with available thermal energy is generally insufficient to produce an effect. It should be remembered however, that in many clinical procedures exposure regimes are such 
that the retinal effects will have contributions from both thermal and photochemical components and in general these two mechanisms will enhance each other.

\section{Free Radicals}

Although the exact biochemical mechanisms remain obscure, there is now increasing evidence to suggest that both photochemical damage and ageing are induced by a common process, the toxic effects of free radicals. These are very short-lived molecular fragments that have unpaired electrons in their outer orbit and because of this unstable structure they are very reactive and very toxic. Free radicals are produced by metabolic processes involving oxygen and by the action of light. We have therefore two paradoxes; oxygen is essential for life but toxic and light is essential for vision but toxic. The retina has one of the highest metabolic demands of any tissue in the body, thus the combination of light and oxygen greatly enhances the portability of deleterious reactions within its component cells.

Within tissues, molecules that absorb photons are called molecular sensitisers or photosensitisers. On absorbing a photon they assume an excited electronic state, called the singlet or S1 state. However, such an excited condition is extremely short-lived and usually lasts less than $10^{-5} \mathrm{~s}$. There are three ways in which the S1 state can lose its absorbed energy: (l) it can interact directly with the surrounding solvent, usually water; (2) it can emit a lower energy photon, a process known as fluorescence; and (3) it can undergo a radiationless transition into a metastable or triplet state, designated ${ }^{3} \mathrm{~S}$.

The triplet states are more stable and longerlived than the S1 states and therefore have time to react with other molecules. This excited state is thought to be the common initial step in the pathway of most photochemical procedures, but further reactions are divided into two major types. Type I reactions are those in which the ${ }^{3} \mathrm{~S}$ interacts directly with the surrounding medium to produce radicals. Type II reactions are those in which the ${ }^{3} \mathrm{~S}$ interacts with molecular oxygen to produce singlet oxygen $\left({ }^{1} \mathrm{O}\right)$ or superoxide $\left(\mathrm{O}^{-\mathrm{O}}\right)$ radicals.

In both cases the resultant free radicals can and do attack other molecules. The most destructive of these attacks is that of singlet oxygen upon an essential component of biological membranes, the polyunsaturated fatty acids. The single oxygen radicals cause peroxidation of the polyunsaturated fatty acids and in doing so change the characteristics of the affected cell membrane, in some instances so adversely that it may subsequently degenerate.

To prevent the ravages of such destructive chemical moieties, cells have evolved a variety of defence mechanisms in both dividing and non dividing systems.

Dividing cells represent the ultimate solution to such attacks by continuously replacing their DNA, cellular constituents, and cell membranes. The toxic products of free radicals are thereby prevented from accumulating to such levels that the subject cell could be compromised. To some extent this explains the presence of such systems in cellular locations where sustained exposure to optical radiation could be expected, eg. the skin and the corneal epithelium and in situations where high concentrations of oxygen are found, eg. the blood. In extreme climatic conditions of high sunlight the dividing cells found in the epidermis of the skin acquires a further protective component in the accumulation of melanin, a pigment which both prevents the deeper penetration of optical radiation and at the same time acts as a free radical scavenger or sink, thereby neutralising their toxic effects. Hence the distribution of black races in geographic locations of high terrestrial levels of solar optical radiation.

Non dividing cells are totally dependent upon endogenous, chemically mediated defence systems. The pigment epithelium is an exception in containing the free radical scavenger melanin, but many contain such membrane-stabilising compounds as taurine or Vitamin E. All cells contain enzyme systems that minimise the effects of free radicals, such as superoxide dismutase, peroxidase or catalase and non dividing cells in exposed positions have such protective mechanisms extensively developed. The pigment epithelium is a prime example of a non-dividing cell constantly exposed to free radical generation, both by light and also by degradation of the phagocytosed light sensitive disc membranes of the photoreceptor cells.

To encapsulate this section with the example of retinal photoreceptors we have the following considerations: 
(1) The photoreceptor cells are at risk throughout life from the effects of free radicals due to the combined exposure to light and oxygen.

(2) They derive short-term protection from the presence of membrane-stabilising agents such as taurine and Vitamin E.

(3) Their long-term protection is secured by the continuous replacement of their lightsensitive membranes. Experimental evidence suggests that there is a difference in replacement rates between rods and cones such that under stress the cone system breaks down before that of the rods.

(4) The long-term preservation of photoreceptor cells is also afforded by the free radical neutralising effects of both melanin and the well-developed enzymic battery within the adjacent retinal pigment epithelial cells.

(5) In situations where high retinal irradiances occur or where exposures are continued over a long period of time the above protective mechanisms may be overwhelmed.

(6) The protective mechanisms decrease in effectiveness with increasing age.

\section{Clinical Uses of Photochemical Damage}

Few experiments have been conducted using photochemical effects of lasers to treat ophthalmic conditions. The only procedures that have gone into limited clinical investigation have been those involving the injection of a photosensitising agent Hematoporphorin derivative (HPD) in cases where malignant melanomas of the choroid are present. The theoretical concept behind this treatment is that the HPD is accumulated at higher levels in tumour cells than in surrounding non-malignant cells. After systemic injection of dye, the tumour is then irradiated at sub-thermal levels of irradiation, preferably with the dye laser system tuned to the peak absorption of HPD. Theoretically the laser radiation induces fluorescence within the HPD, the fluorescence is associated with the generation of free radicals and as this generation only occurs within tumour cells and as such agents are highly cytotoxic the tumour cells are killed. Initial trials in dermatology showed some promise for this technique but in ophthalmology the results are not very impressive. A theoretical development of this technique however, holds a great deal of promise in that if monoclonal antibodies are developed to specific retinal cell systems and the monoclonals are tagged, either with chromophores or fluorophores, then using this system in conjunction with low energy dye laser irradiation, a highly specific photochemical action spectra may be developed to selectively destroy individual cell populations.

In clinical practice photochemical damage may account for the tritanopic colour defect seen after panretinal ablation in diabetics. The need to avoid repetitive exposure to short wavelength laser sources is now of great importance as a recent study of the blue cone sensitivity of ophthalmic laser surgeons immediately after performing panretinal ablation show a suppression of blue cone sensitivity lasting for four to six hours. This phenomenon occurs as a result of reflections of the laser's aiming beam coming back into the operator's eye from the surface of the contact lens. In these circumstances the retinal irradiance of the surgeon may be about $40-90 \mu \mathrm{W} / \mathrm{cm}^{2}$ a figure that approaches that known to cause photochemical damage in repetitive exposures in monkeys. In experienced ophthalmic laser surgeons the suppression of blue cone sensitivity becomes irreversible.

\section{Photoablation}

In the early eighties a new class of lasers was seen called excimer lasers. Excimer is an acronym derived from the first two and last syllables of the words that describe the physical state of the lasing media used in such lasers, excited dimers. Excited dimers are two atoms of an inert gas bound in a highly excited state with atoms of a halogen to form a temporary highly unstable association as a diatomic rare gas halide. The decay of these unstable molecules is accompanied by the emission of a highly energetic photon of ultraviolet light. The wavelength of the photons and therefore the wavelength of the light emitted from an excimer laser is dependent upon the particular gas mixture used to fill the laser cavity. For example, the emission from a mixture of argon and fluorine occurs at $193 \mathrm{~nm}$ and that for krypton fluoride at $248 \mathrm{~nm}$. These lasers emit their radiation as a train of individual pulses whose duration is typically about 10 nanoseconds long and with a selectable pulse repetition frequency of anywhere between 1 and 100 pulses per 
second. Typical pulse energies of excimer laser vary from 20 to $200 \mathrm{~mJ}$.

In the materials and electronics industries excimers are used to etch submicron patterns onto the surface of plastics and other polymers with no degradation processes being conducted to unirradiated areas. In this situation, spatial resolution can be considered in terms of width and depth of material removed with each pulse of laser energy. In theory the very best lateral resolution would occur if only those molecules directly irradiated were removed and the best depth resolution would be one layer of molecules removed per laser pulse. In practice more than one layer of molecules is removed with each pulse because photons penetrate through the surface of the target. As previously stated the penetration depth is a function of the nature of the target material and the wavelength of the incident of radiation. In biological tissue minimum penetration occurs at two wavelengths, $190 \mathrm{~nm}$ in the ultraviolet $\mathrm{C}$ and $2.9 \mu \mathrm{m}$ in the infrared $\mathrm{C}$. In the former a penetration depth of 1 to $5 \mu \mathrm{m}$ occurs as a result of absorption of the UV photons by macromolecules and in particular, proteins. In contrast an absorption depth of 20 to $25 \mu \mathrm{m}$ is observed at $2.9 \mu \mathrm{m}$ and in this case results from absorption of infrared photons by water molecules. These respective penetration depths define the maximum amount of target tissue that will be directly affected with each pulse of laser radiation.

There are currently two theories on the removal of tissue by absorbed photons in the ultraviolet. The first of these suggests that the photons cause ultrafast tissue heating, so-called photon-phonon interactions, and that the vibration of superheated molecules causes them to fall apart. The second theory asserts that the target reaction is not thermal but photon induced molecular decompensation, or photoablation. The protagonists of this theory suggest that molecules in the target are released as a direct result of rupture of intramolecular bonds whose uncoupling is induced by the absorption of high energy ultraviolet photons. Such a mechanism is possible because in the ultraviolet at $193 \mathrm{~nm}$ individual photons have an energy in excess of six electron volts and are capable of disrupting valency electrons. As the molecules break up the components fragments require a greater space than the original molecule. Fragment expansion can only be facilitated by displacement and as these processes are only occurring at or close to the target surface, such displacement inevitably results in the loss of material from the exposed surface.

In practice, the depth of tissue removed per pulse is determined by the pulse energy, with a maximum set by the penetration depth. At 193 $\mathrm{nm}$ varying the pulse energy means that the predetermined depths of tissue can be removed at any level between 1/40 and $1 \mu \mathrm{m}$ per pulse.

The unique nature of the photoablation process becomes even more apparent when excimers are used to incise soft tissue such as the cornea. In such experiments it was apparent that the walls of the incision were perfectly smooth and coated by a pseudomembrane rather like a "shrink-wrap" or "clingfilm" covering used for foodstuffs. This pseudomembrane is thought to arise from the somewhat random recombination of the ruptured bonds of the molecular fragments that remain locked into the tissue matrix of the walls and floor of the excision. Whatever its origin, it creates an optically smooth surface and imparts a limited osmotic integrity to the cut surface. The second quality of excimer incisions is that no damage is apparent in the walls of the excision outside a zone between 100 to $300 \mathrm{~nm}$ in extent. This lack of secondary or conductive damage is unique to the excimer lesion.

With such precise control of tissue removal and with such a clean and highly localised wound an excimer seems an ideal instrument to avoid the technical limitations incurred during radial keratomy procedures carried out with steel or diamond knives. ${ }^{5} \mathrm{~A}$ great deal of interest has been expressed also in the possibility of using excimer systems to make excisions to correct astigmatism induced by cataract surgery.

One further attribute of the excimer is that the beams are usually of rectangular cross section and typically $20 \mathrm{~mm} \times 10 \mathrm{~mm}$. The beam cross section can be made square by passing it through a cylindrical lens and then circular by passing it through an aperture. If an excimer beam of circular cross section and say $10 \mathrm{~mm}$ in diameter is allowed to fall upon a biological surface, then at a given pulse energy a $10 \mathrm{~mm}$ diameter disk $1 \mu \mathrm{m}$ deep will be ablated with each laser pulse. ${ }^{6}$ If the tissue was the cornea then a perfectly round smooth surface excavation would be created to a predetermined depth and this is 
precisely the configuration desired for preparing the recipient eye in corneal graft procedures. Similarly, if instead of using a beam of circular cross section one was obtained which was an annulus, then the corneal button to be grafted could also be cut from the donor eye and the bed and button would be obtained by using a combination of prisms and lenses called an axicon.

The facility for producing large beams of circular cross section has also profound implications for refractive surgery. If for example a beam was produced in which the energy distribution across it was varied in a controlled fashion, the beam would be used to sculpture or shape the surface of the target. For example, if most of the energy were concentrated at the centre and it was slowly reduced towards the periphery, then the resultant excavation would be concaved or effectively be a negative lens. In contrast, if most of the energy were concentrated in an annulus around the edge and decreased as it approached the centre, then the resultant excavation would be convexed or a positive lens.

In practice, it is easier to control energy in time rather than in space. If for example, an iris diaphragm is placed concentric with a laser beam and then progressively opened or closed with a given number of laser pulses being directed through the aperture in the diaphragm at each aperture size, then the target surface will be ablated to give rise to a series of concentric circular steps. If the aperture is motorised and suitably integrated with the laser control system, then the steps could become infinitely small and thus in practice a smooth curve would be generated. This is the principle currently employed in commercial systems designed to carry out this sort of surgery.

Computer predictions show that for a $3 \mathrm{~mm}$ disc a 10 dioptre correction could be induced, removing maximally only $25 \mu \mathrm{m}$ of stromal material. Subsequent experiments on animals have shown these predictions to be correct in terms of refractive power of the initially ablated surface.

Providing ablations are executed with suitable laser exposure parameters and providing they are shallow, ie. less than $50 \mu \mathrm{m}$ in depth, then scar tissue and loss of transparency have not been found to be significant problems in eighteen month follow-up studies in monkeys. Exposures in which the entire volume of ablated material was restricted to Bowman's layer were particularly notable for the virtual absence of scar collagen and in such cases the initial curvature of the ablated surface of collagen seemed to remain unchanged.

In deeper exposures where stromal collagen restructuring was apparent the new collagen formation followed the new radius of curvature of the ablated surface and therefore the implications are that the refractive change should remain relatively stable within limits.

Although concern has been expressed in relation to the mutagenic effects of ultraviolet radiation used for this sort of surgery, a number of laboratories studying this have not found any mutagenic effect associated with radiation at 193 $\mathrm{nm}$. This is not the case for 248 where significant chromosomal abnormalities have been identified, and another excimer laser wavelength of $308 \mathrm{~nm}$ has been shown to induce cataract formation.

A further procedure utilising the excimer is the excision of small troughs directly through the sclera of the eye down towards the trabecular meshwork. In this procedure the laser is aimed towards the canal of Schlemm and then with repeated pulses tissue is excised from the sclera, $1 \mu \mathrm{m}$ per pulse. As soon as the surgical end point is realised, ie. the outflow of aqueous, then the laser begins to expend its energy ablating aqueous rather than scleral or other tissues. In this glaucoma procedure then the surgery is totally self-limiting and effectively ceases the moment the surgical end point is achieved.

Future potential uses of photoablative mechanisms is the possible use of fibre optics with excimer lasers. Currently fibre optic transmission systems in the ultraviolent are very poor and performance decreases with decreasing wavelength. To date some workers have utilised a $308 \mathrm{~nm}$ emission line of excimer together with fibre optics to experimentally ablate the lens. The concept behind this surgery is that a very small hole is made in the cornea or in the limbus of the eye through which is passed a tube. The outer portion of the tube acts as an aspiration system and in the inner portion of the tube is a fibre optic. Successive laser pulses are passed down the fibre optic and cause ablation and fragmentation of the lens and the ablated material is aspirated. Developments of procedures like this together with folding or inflatable intraocular 
lenses means that very small incisions can be utilised. In general, the smaller the initial incision for cataract surgery, then the less the post operative complications of astigmatism.

Recently a number of infrared lasers (hydrogen fluoride, erbium YAG, colour centre lasers and Raman lasers) with emission wavelengths around $2.9 \mu \mathrm{m}$ have been used experimentally for both making incisions in the cornea and in conjunction with fibre optics for cutting vitreous strands. At $2.9 \mu \mathrm{m}$ the emission wavelength coincides with the absorption peak of water. At this wavelength radiation only penetrates between 20 and $25 \mu \mathrm{m}$ into the surface of tissues. The confined absorption depth, coupled with a short pulse duration and a relatively high peak power means that the ensuing ultrafast thermal effects do not result in significant conductive damage to adjacent tissues. Under worse case conditions this conductive damage is limited to about $25 \mu \mathrm{m}$ on either side of the induced wound. Again this sort of focal effect associated with a laser capable of incising tissue has very good indications for its potential usefulness in ophthalmic surgery.

Laser technology moves at an extremely fast rate which is driven by investment and research in the opto-electronics industry together with massive financial investments from military agencies. The resulting increase in availability of lasers with new pulse parameters and new wavelengths mean that each year a number of laser systems are developed that may have clinical potential. In order to optimise treatment regimes these systems need to be constantly reviewed and the biological database developed. One of the major problems in relation to the interaction of laser light and tissue is that biological action spectra tend to have sharp peaks, and small changes in wavelength for sound surgical reasons may result in totally undesirable reactions due to unsuspected absorbing systems within the target. However, even given these constraints on new development the results of laser research in relation to ophthalmology over the last 25 years have resulted in an incredible range of surgical lasers which have given the ophthalmologists the ability to carry out techniques that no-one could have predicted before the dawn of lasers.

\section{References}

${ }^{1}$ Zaret MM, Breinin GM, Schmidt H, Ripps H, Siegel IM, Solon LR. Ocular lesions produced by an optical maser (laser). Science 1961; 134: 1525-6.

${ }^{2}$ Curtin TL and Boyden DG. Reflected laser beam causing accidental burn of the retina. Am J Ophthalmol 1968; 65: 188-9.

${ }^{3}$ Mainster MA, Sliney DH, Belcher DC, Buzney MS. Laser Photodisrupters: Damage mechanisms, instrument design and safety. Ophthalmology 1983; 90: 973-91.

${ }^{4}$ Fankhauser F, Lortscher H, Van der Zypen E. Clinical studies on high and low power radiation on some structures of the anterior and posterior segments of the eye, experiences in the treatment of some pathological conditions of the anterior and posterior segments of the human eye by means of Neodymium YAG laser driven at various power levels. Int Ophthalmol 1982; 5: 15-32.

${ }^{5}$ Marshall J, Trokel S, Rothery S, Schubert H. An ultrastructural study of corneal incisions induced by an excimer laser at $193 \mathrm{~nm}$. Ophthalmology 1985; 92: 749-58.

${ }^{6}$ Marshall J, Trokel S, Krueger R. Photoablative reprofiling of the cornea using an excimer laser: Photorefractive keratectomy. Lasers in Ophthalmology 1986; 1: 21-48. 